\title{
Empathy in Undergraduate Medical Students of Bangladesh: Psychometric Analysis and Differences by Gender, Academic Year, and Specialty Preferences
}

\author{
Asma Mostafa, ${ }^{1}$ Rozina Hoque, ${ }^{2}$ Mohammad Mostafa, ${ }^{3}$ \\ Md. Mashud Rana, ${ }^{4}$ and Faisal Mostafa ${ }^{5}$ \\ ${ }^{1}$ Department of Anatomy, Chattagram Maa-O-Shishu Hospital Medical College, Chittagong, Bangladesh \\ ${ }^{2}$ Department of Pharmacology \& Therapeutics, Chattagram Maa-O-Shishu Hospital Medical College, Chittagong, Bangladesh \\ ${ }^{3}$ Department of Psychiatry, Chattagram Maa-O-Shishu Hospital Medical College, Chittagong, Bangladesh \\ ${ }^{4}$ Department of Pharmacology \& Therapeutics, Chittagong Medical College, Chittagong, Bangladesh \\ ${ }^{5}$ Department of Surgery, Diabetic Association Medical College, Faridpur, Bangladesh
}

Correspondence should be addressed to Asma Mostafa; asmamostafaisrat@yahoo.com

Received 11 February 2014; Accepted 18 March 2014; Published 7 April 2014

Academic Editors: M. V. Seeman and A. Weizman

Copyright (C) 2014 Asma Mostafa et al. This is an open access article distributed under the Creative Commons Attribution License, which permits unrestricted use, distribution, and reproduction in any medium, provided the original work is properly cited.

\begin{abstract}
Empathy is considered to be associated with better patient compliance, satisfaction, and clinical outcomes. The aim of the study is to measure and examine empathy among a sample of undergraduate medical students of Bangladesh. It was a cross-sectional study and all the medical students of first through fifth year enrolled at Chattagram Maa-O-Shishu Hospital Medical College during the study period of 2014 were surveyed. Participants anonymously completed the Jefferson Scale of Empathy Medical Student version translated into Bengali language, a valid and reliable 20 -item self-administered questionnaire. Principal component factor analysis with varimax rotation and Cronbach's alpha coefficient were calculated to check validity and reliability of the scale. ANOVA was used to examine the differences in empathy between gender, academic years, and specialty preferences. The mean empathy score was $110.41 \pm 13.59$. Cronbach's alpha coefficient was 0.88 . There were significant associations between gender and empathy scores. The level of empathy in medical students gradually increases after clinical training in medical college. A nonsignificant difference was found between empathy scores and specialty preferences. It is suggested that the medical curriculum in Bangladesh should include more extensive program to promote empathy and other humanistic values among the medical students.
\end{abstract}

\section{Introduction}

Empathy is the ability to understand and share the feelings of others [1]. It is the power of entering into others' personality and imaginatively experiencing their emotional state. Hojat et al. define empathy as "a predominantly cognitive attribute that involves understanding of the patient's experiences, concerns, and perspectives, and a capability to communicate this understanding. An intention to help by preventing and alleviating pain and suffering is an additional feature of empathy in the context of patient care" [2]. Empathy is essential in "physician-patient relationship" to produce a desire within physician to help the patient, to know what the patient is thinking or feeling, to provide best care to the patient, and to blur the line between physician and patient [3]. Empathy is important in development of interpersonal understanding which allows the patient to feel respected and validated [3]. It is the key element which can propel the physician toward altruistic action [4]. It helps the physician to be closer to patient, putting the benefit of other above those of self, even at some sacrifice to one self. Empathy is considered to be associated with improved health outcomes. A good physician-patient relationship is capable of creating better patient compliance, satisfaction, and clinical outcomes [5].

There are various factors determining empathy, such as, age, gender, family background, culture, intelligence, emotional stability, and education [6,7]. The study of 
empathy in patient care is important not only within a society but also among different cultures because of variations in medical education curriculum, cultural norms, and social learning.

One of the major tasks in medical education is to maintain and increase empathy in medical students for patients. But various researches suggest that empathy in medical students decreases during the course of medical training [8-10]. Students experience medical training as stressful which might be injuring instead of fostering empathy. [11]. During this training, students also learn how to manage the stresses and anxiety of illness which may develop maladaptive responses that lead to a decline in their level of empathy [12]. Besides this, dependence on technology for diagnosis and limited interactions with patients may lead to a decrease in empathy among the medical students [13].

Empathy is believed to be measurable and teachable. The Association of American Medical Colleges recommended that empathy should be integrated and assessed in medical education [2, 14]. Various research instruments, for example, Interpersonal Reactivity Index, Balanced Emotional Empathy Scale, and Jefferson Scale of Empathy (JSE), are available to measure empathy [11]. Among the self-reported instruments, JSE was used by various researchers to measure empathy specifically within the context of the physicianpatient relationship $[7,15-20]$. The validity and reliability of the JSE have been also reported [18, 21].

Exercises and a more extensive program have been introduced formally in various medical curricula worldwide, to asses and promote empathy and other humanistic values among the medical students [12]. Such programs are still not well established in Bangladesh. The present study was designed to examine and measure empathy among a sample of undergraduate medical students of Bangladesh and also to compare the effects of gender, academic year, and specialty preferences on empathy.

\section{Method}

The study was cross-sectional in nature and carried out in Chattagram Maa-O-Shishu Hospital Medical College (CMOSHMC), Chittagong, Bangladesh, during the study period of 2014. The study was approved by the CMOSHMC Ethical Committee.

2.1. Participants. All medical students of first through fifth year enrolled at CMOSHMC during 2014 were eligible to participate in the study. Participants included 426 medical students from five academic years, in the first $(N=100)$, second $(N=88)$, third $(N=86)$, fourth $(N=77)$, and fifth year $(N=75)$. There were 291 female and 135 male students in the study population. The undergraduate medical curriculum in Bangladesh is a traditional five year medical school training with 1.5 years preclinical study, 2 years paraclinical study with limited patient contact, and 1.5 years clinical study.
2.2. Instruments. The JSE has been adapted to several countries and languages $[7,15-18,20]$. It exists in three versions, HP-Version: for administration to physicians, S-Version: for administration to medical students, HPS-Version: for administration to students in other health professions like nursing, paramedical courses [2]. In this study, the Jefferson Scale of Empathy Medical Student version (JSE S-version) translated into Bengali language was used to measure medical students' attitudes toward empathic physician-patient engagement in the contact of patient care. The JSE S-version was translated into Bengali language using a back translation procedure to ensure the accuracy of the translation $[2,7$, 15-20, 22, 23]. At first, JSE S-version was translated from English into Bengali language by two bilingual researchers having a detailed understanding of the instrument. Later the translated Bengali version was sent to another three bilingual researchers who had not seen the original English version and were asked to translate Bengali version back into English. Then the original English version was compared with the back-translated English versions to see consistency and adaptations that were done in wording, where needed, to make the text consistent with Bengali culture without losing intended key concepts, and lastly a final Bengali version was produced. The JSE S-version includes 20 items each answered on a seven-point Likert scale. 10 positively worded items were linked to "perspective taking" and directly scored ( $1=$ strongly disagree, 7 = strongly agree). 10 items were negatively worded and reversed scored ( $1=$ strongly agree, $7=$ strongly disagree) [13]. Eight of the ten negatively worded items were concerned with "compassionate care" and 2 items were linked to "standing in the patient's shoes." Scores ranged from 20 to 140. Higher values indicate a higher degree of empathy [11].

Specialties were categorized into three groups like peopleoriented, technology-oriented, and other specialties, as shown in Table $1[2,10,23]$. Students specified their career specialty intentions, in terms of possibility of entering each of the specialties.

2.3. Procedures. Before going into the survey, it was explained to the participants that the questionnaire was about empathy asking for their personal beliefs and opinions and that the results would be used for research purpose. The participants were also informed that the study would be anonymous, so it would not be possible to identify them anywhere in the questionnaire. Therefore, there was no need to answer any questions in a manner that would be viewed as a "good behavior" by others. It was also stated that participation was voluntary but returning the questionnaire would be taken as consent to participate. There would be neither reward for participating nor any penalty for not participating in the survey. Respondents did not need to sign their names, but they were asked to provide information on their gender and academic year. JSE S-version in Bengali questionnaire was distributed to the 1st, 2nd, 3rd, 4th, and 5th year medical students during their regular classes. It has been suggested that printed copies command greater response rates over electronic versions [24]. Participants were given 1 day to complete the study and also asked to take the test individually. 
TABLE 1: Career preference categories.

\begin{tabular}{|c|c|c|}
\hline People-oriented specialties & Technology-oriented specialties & Other specialties \\
\hline $\begin{array}{l}\text { Internal medicine and medicine } \\
\text { subspecialties: cardiology, } \\
\text { neuromedicine, nephrology, } \\
\text { endocrinology, and } \\
\text { gastroenterology }\end{array}$ & $\begin{array}{l}\text { General surgery and surgical subspecialties: } \\
\text { ophthalmology, orthopaedics surgery, urology, } \\
\text { neurosurgery, plastic surgery, ENT, and pediatric } \\
\text { surgery }\end{array}$ & $\begin{array}{l}\text { Pharmacology/forensic } \\
\text { medicine/community } \\
\text { medicine/anatomy/physiology }\end{array}$ \\
\hline Psychiatry/dermatology & $\begin{array}{l}\text { Anesthesiology/radiology/physical } \\
\text { medicine/oncology }\end{array}$ & Undecided \\
\hline Pediatrics & Obstetrics and gynecology & \\
\hline General practitioner & Pathology/microbiology/hematology/biochemistry & \\
\hline
\end{tabular}

2.4. Statistical Analysis. Questionnaires missing information on five or more items were considered incomplete and excluded from the data for subsequent analysis [2]. If a respondent fails to answer 4 or fewer items, the missing values were replaced with the mean score calculated from the items the respondent completed [2]. After data collection, frequency distributions, central tendencies, and dispersions of the Bengali version of JSE S-version scores were determined. Cronbach's alpha coefficient was calculated to assess the internal consistency aspect of reliability of the instrument. A reliability of 0.70 and higher was considered satisfactory [7, $14,20,25]$. Correlation between each item and the total score (item-score correlation) was calculated. Principal component factor analysis with varimax rotation was used to search for the underlying factor structure of the Bengali version of the JSE S-version. Eigenvalues greater than 1 were required to retain factors [26] and factor loadings of 0.35 or greater were required for the interpretation of the factor structure [14]. ANOVA with post hoc test analysis was used to examine the differences of empathy scores related to gender, academic years, and specialty preferences. Data were analyzed using SPSS version 11.5 and a $P$ value $<0.05$ was set as statistically significant.

\section{Results}

348 questionnaires were returned out of possible 426 . The overall response rate was $81.69 \%$. Table 2 shows that first year students have the highest response rate and fifth year students have the lowest response rate.

Range, mean $\pm \mathrm{SD}$, and quartile points of the Bengali version of JSE S-version empathy scores are shown in Table 3. Cronbach's alpha coefficient is in an acceptable range which indicates that Bengali version of JSE S-version was internally consistent for psychological measures.

The Kaiser-Meyer-Olkin (KMO) analysis was performed, yielding an index of 0.89 . The result for Bartlett's test of sphericity was 2036.40 and was highly significant $(P=0)$. This information indicates the appropriateness of principal components analysis. Summary results of principal component factor extraction with varimax rotation of data for the 20 items of the Bengali version of JSE S-version are shown in Table 4 . Three factors with eigenvalue $>1$ emerged and accounted for a total of $44.80 \%$ of the variance. Factor 1 loaded seven items having a factor coefficient of greater than
TABLE 2: Socio-demographic characteristics of the participants.

\begin{tabular}{lccc}
\hline Characteristics & $\begin{array}{c}\text { Number of } \\
\text { students }\end{array}$ & $\begin{array}{c}\text { Number of } \\
\text { responders }\end{array}$ & $\begin{array}{c}\text { Response rate } \\
(\%)\end{array}$ \\
\hline Academic Year & & & \\
1st & 100 & 98 & 98 \\
2nd & 88 & 82 & 93.18 \\
3rd & 86 & 59 & 68.60 \\
4th & 77 & 69 & 89.61 \\
5th & 75 & 40 & 53.33 \\
Gender & & & \\
Male & 135 & 104 & 77.04 \\
Female & 291 & 244 & 83.85 \\
\hline
\end{tabular}

TABLE 3: Descriptive statistics for the Bengali version of the JSPE-S.

\begin{tabular}{lc}
\hline Statistics & Value \\
\hline Range & $69.00-135.00$ \\
Mean \pm SD & $110.41 \pm 13.59$ \\
25th percentile & 100.00 \\
50th percentile & 114.00 \\
75th percentile & 121.00 \\
Reliability (Cronbach's alpha coefficient) & 0.88 \\
\hline
\end{tabular}

$N=348$.

0.35 , five items related to "perspective taking" and two items related to "compassionate care." Factor 2 loaded eight items with a factor coefficient of greater than 0.35 for statements reverse-scored, six items related to "compassionate care" and two items related to "standing in the patient's shoes." Factor 3 loaded with five items with a factor coefficient of greater than 0.35 related to "perspective taking."

Table 4 also shows the mean $\pm \mathrm{SD}$, communalities (h2), and corrected item-total correlations for each item in the Bengali version of JSE S-version. The mean item score responses ranged from 4.14 to 6.39 . The lowest score was observed for item 18 (reverse score) and the highest score was for item 2. These indicate that the students' responses tend to be skewed towards the upper end of the scale. The interitem score correlation was positive. Corrected item-total score correlation ranged from a low of 0.334 for item 8 (reverse score) to a high of 0.657 for item 18 (reverse score). 
TABLE 4: Principal component analysis with varimax rotation and corrected item-total correlations of the Bengali version of JSE S-version.

\begin{tabular}{|c|c|c|c|c|c|c|}
\hline Items & $F 1$ & $F 2$ & $F 3$ & Mean \pm SD & Communalities $h 2$ & Corrected item-total correlations $r_{\mathrm{i}-\mathrm{t}}$ \\
\hline 2 & 0.720 & 0 & 0 & $6.39 \pm 0.75$ & 0.572 & 0.556 \\
\hline $1 \mathrm{R}^{*}$ & 0.718 & 0 & 0 & $5.04 \pm 1.76$ & 0.656 & 0.649 \\
\hline 20 & 0.686 & 0 & 0 & $6.17 \pm 0.89$ & 0.591 & 0.562 \\
\hline $19 \mathrm{R}$ & 0.671 & 0 & 0 & $5.89 \pm 1.40$ & 0.476 & 0.418 \\
\hline 4 & 0.424 & 0 & 0 & $6.01 \pm 0.87$ & 0.297 & 0.456 \\
\hline 10 & 0.423 & 0 & 0 & $6.06 \pm 0.88$ & 0.262 & 0.422 \\
\hline 13 & 0.382 & 0 & 0 & $5.82 \pm 0.94$ & 0.206 & 0.361 \\
\hline $6 \mathrm{R}$ & 0 & 0.721 & 0 & $4.25 \pm 1.48$ & 0.635 & 0.646 \\
\hline $18 \mathrm{R}$ & 0 & 0.694 & 0 & $4.14 \pm 1.76$ & 0.612 & 0.657 \\
\hline $3 \mathrm{R}$ & 0 & 0.658 & 0 & $4.72 \pm 1.33$ & 0.442 & 0.428 \\
\hline $14 \mathrm{R}$ & 0 & 0.652 & 0 & $4.93 \pm 1.66$ & 0.481 & 0.528 \\
\hline $7 \mathrm{R}$ & 0 & 0.528 & 0 & $5.46 \pm 1.42$ & 0.430 & 0.561 \\
\hline $8 \mathrm{R}$ & 0 & 0.520 & 0 & $5.00 \pm 1.36$ & 0.325 & 0.334 \\
\hline $12 \mathrm{R}$ & 0 & 0.494 & 0 & $5.68 \pm 1.16$ & 0.297 & 0.421 \\
\hline $11 \mathrm{R}$ & 0 & 0.407 & 0 & $6.06 \pm 1.16$ & 0.360 & 0.533 \\
\hline 16 & 0 & 0 & 0.698 & $5.68 \pm 0.90$ & 0.531 & 0.386 \\
\hline 17 & 0 & 0 & 0.638 & $5.47 \pm 1.16$ & 0.474 & 0.436 \\
\hline 9 & 0 & 0 & 0.560 & $5.83 \pm 1.13$ & 0.495 & 0.508 \\
\hline 15 & 0 & 0 & 0.553 & $5.83 \pm 1.07$ & 0.369 & 0.408 \\
\hline 5 & 0 & 0 & 0.487 & $5.98 \pm 1.02$ & 0.449 & 0.437 \\
\hline$\%$ of variance & 31.29 & 7.46 & 6.05 & & & \\
\hline Alpha & 0.75 & 0.81 & 0.68 & & & \\
\hline
\end{tabular}

${ }^{*} \mathrm{R}$ : items were reverse scored.

The factor pattern coefficients of 0.35 and below were replaced by zeros.

Table 5 shows a statistically highly significant difference in mean empathy scores between female and male medical students. Figure 1 shows that female medical students have higher empathy scores than male medical students of five academic years. ANOVA shows that gender had no effect on empathy score for medical students in first, third, and fifth years with $P$ values $0.17,0.11$, and 0.40 , respectively, but revealed a significant difference in fourth year medical students $(P=0.01)$ and nearly significant difference in second year medical students $(P=0.07)$.

Analysis of variance showed that there was a significant difference between mean scores from various academic years (Table 5). In addition, post hoc analysis showed a significant difference between second year and first $(P=0.02)$, third $(P=0.04)$, fourth $(P=0)$, and fifth years $(P=0)$. There was also a significant difference between third year and fourth year $(P=0.01)$. No difference is seen between fourth and fifth year students $(P=0.63)$. Figure 2 shows that there is a decline in mean empathy scores from first to second year, followed by a gradual increase from third to fourth year. Although there is a decrease of mean empathy scores from fourth to fifth year, it was statistically nonsignificant.
A nonsignificant difference was found between medical students preferring "people-oriented" or "technologyoriented" or "other" specialties.

\section{Discussion}

The aim of this study was to examine levels of patient empathy in undergraduate medical students of Bangladesh. In Bangladesh there is no scale to examine empathy in medical students. In the present study, Bengali version of JSE S-version was adapted for Bangladeshi population. The reliability of Bengali version of JSE S-version was examined. Cronbach's alpha coefficient in this study $(r=0.88)$ was similar to those reported for Korean $(r=0.84)$ [16], Japanese $(r=0.80)$ [15], Chinese $(r=0.83)$ [22], and South African medical students $(r=0.79)$ [13]. This result indicates that Bengali version of JSE S-version is internally consistent in undergraduate medical students of Bangladesh. The principal component analysis showed a three-factor solution that was somewhat similar to the pattern in other studies. This also provided support for the construct validity of the Bengali version of JSE S-version. 
TABLE 5: Group differences of the Bengali version of JSE S-version.

\begin{tabular}{|c|c|c|c|c|c|}
\hline \multirow{2}{*}{ Group } & \multirow{2}{*}{$N$} & \multirow{2}{*}{ Range } & \multirow{2}{*}{ Mean \pm SD } & \multicolumn{2}{|c|}{ Statistical difference } \\
\hline & & & & $F$ value & $P$ value $^{\dagger}$ \\
\hline \multicolumn{6}{|l|}{ Gender } \\
\hline Male & 104 & $72.00-135.00$ & $106.72 \pm 14.33$ & \multirow{2}{*}{11.28} & \multirow{2}{*}{$0.00(\mathrm{~S})^{\dagger \dagger}$} \\
\hline Female & 244 & $69.00-134.00$ & $111.99 \pm 12.97$ & & \\
\hline \multicolumn{6}{|l|}{ Academic Year } \\
\hline 1 st & 98 & $75.00-132.00$ & $109.77 \pm 15.29$ & \multirow{5}{*}{7.592} & \multirow{5}{*}{$0.00(\mathrm{~S})$} \\
\hline 2nd & 82 & $69.00-133.00$ & $105.02 \pm 14.37$ & & \\
\hline $3 r d$ & 59 & $85.00-129.00$ & $109.75 \pm 11.39$ & & \\
\hline 4th & 69 & $92.00-134.00$ & $115.87 \pm 10.72$ & & \\
\hline 5 th & 40 & $90.00-135.00$ & $114.63 \pm 10.30$ & & \\
\hline \multicolumn{6}{|l|}{ Specialty preferences } \\
\hline People-oriented & 146 & $69.00-133.00$ & $110.45 \pm 13.87$ & \multirow{3}{*}{0.07} & \multirow{3}{*}{$0.93(\mathrm{NS})^{*}$} \\
\hline Technology-oriented & 119 & $72.00-135.00$ & $110.68 \pm 13.28$ & & \\
\hline Other & 83 & $75.00-133.00$ & $109.96 \pm 13.69$ & & \\
\hline
\end{tabular}

${ }^{\dagger}$ Significance of correlation was tested at $5 \%$ level $(P=0.05)$.

${ }^{\dagger \dagger}$ S: significant; ${ }^{*} \mathrm{NS}$ : nonsignificant.

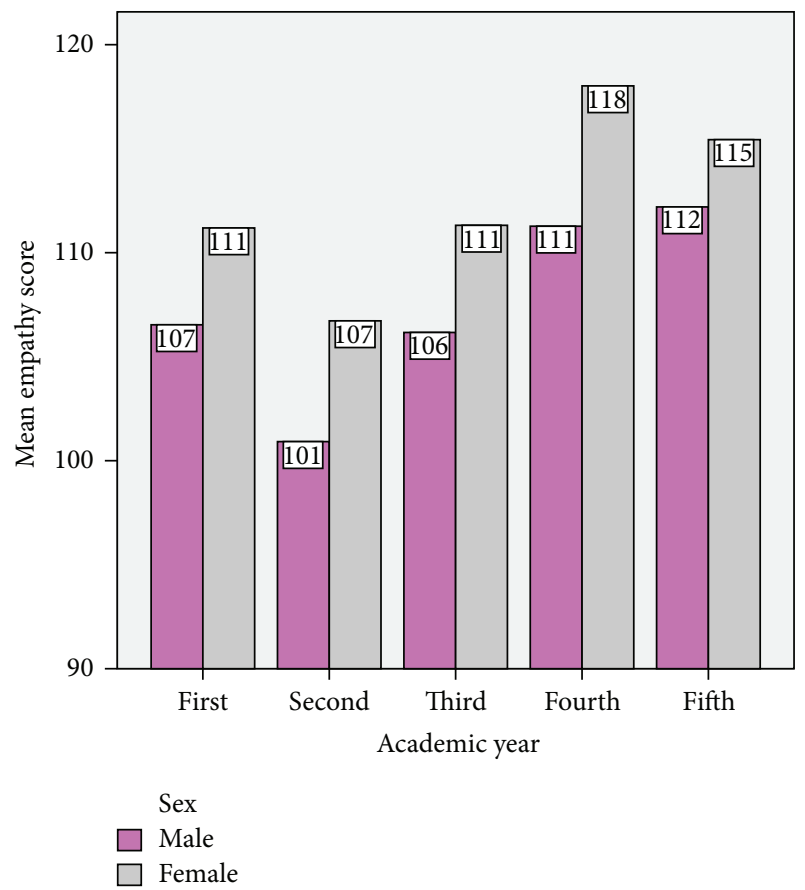

FIgURE 1: Bar diagram showing mean empathy score of medical students in each academic year by gender $(N=348)$.

The mean empathy score of this study (mean $=110.41)$ was similar to Chinese (mean $=109.60)$ [22] and higher than Japanese $($ mean $=104.3)$ [15], Kuwaiti $($ mean $=104.6)$ [27], and Iranian (mean $=105.1$ ) [17] medical students. But the mean score of this study was lower than that of American medical students (mean $=115)$ [8]. It may be due to differences in cultural factors, custom, ethnicity, spiritual belief, educational system, variation of selection

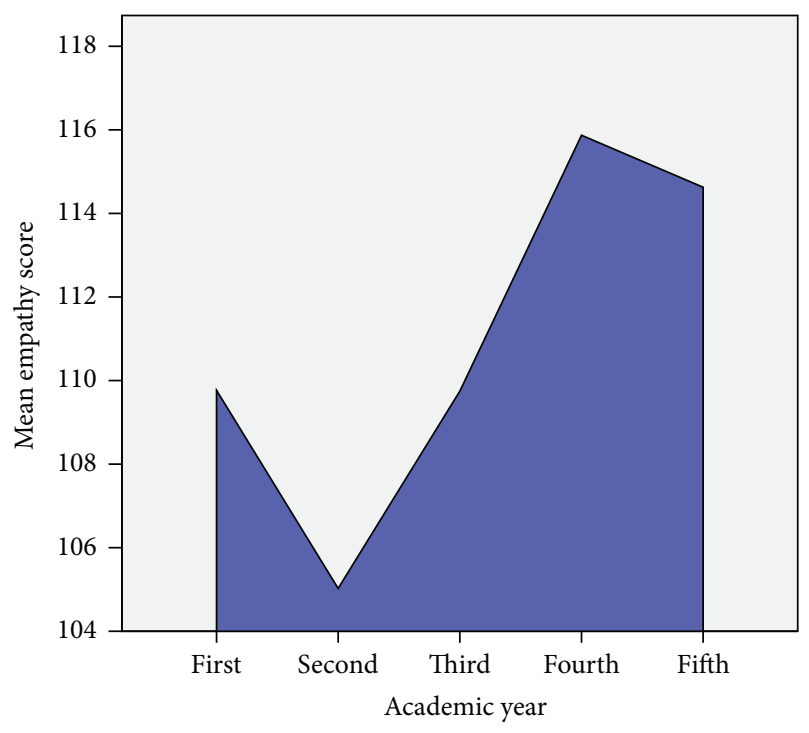

FIGURE 2: Graph showing mean empathy score of medical students in first to fifth academic years $(N=348)$.

of medical students, and availability of appropriate role model.

The gender ratio of entire medical students at CMOSHMC is $68.30 \%$ female to $31.69 \%$ male. This gender ratio will be found in most of medical college in Bangladesh. Previous studies reported that female medical students are more empathic than their male counterparts $[6,10,13,15,16,22,28]$. Finding of this study is also consistent with most of the other studies. The gender difference is due to evolutionary-biological gender characteristics, styles in interpersonal care, socialization, and gender role expectations [13]. Women show a greater understanding of the emotional support which is important to develop interpersonal relationships with patients. 
There was statistically significant difference in empathy scores in different academic years with second year students having the lowest empathy scores. The first year and third year students had statistically significant higher empathy scores than those of second year. This indicates that level of empathy declines in preclinical year followed by a rise of empathy scores in paraclinical year when the students experienced first limited contact with patients. The fourth year had the highest empathy scores followed by a nonsignificant decrease in the fifth year. Although the findings of this study regarding enhancement of empathy are not in agreement with American $[8,10]$ medical students, they are similar to those of Chinese [22], Japanese [15], Korean [16], Portuguese [11], and Kuwaiti [27] medical students. A joint family culture is still predominant in Bangladesh. The age range of the participants of this study is 19 to 24 years. In Bangladesh, person of this age range is usually part of a joint family. They have optimal family support for their living arrangements. So, they can continue their education with less stress and exhaustion. In a study, Kataoka et al. [23] reported that physicians living in joint family are more empathic than those living in a nuclear family. Morling and Lamoreaux [27] have reported that Asians have more "collectivistic and less individualistic social cultures" than Westerners. This may be the reason that empathy scores of the medical students of this study gradually increases after clinical training.

In this study, there was a nonsignificant difference between specialty preferences and empathy scores. However, those students who choose "people-oriented" and "technology-oriented" specialty had slightly higher mean empathy scores than those who preferred "other specialty." Such observations are also reported in Kuwaiti medical students [27]. Students who find themselves to be less comfortable with patients usually choose "technology-oriented" or "other specialty".

This study was cross-sectional in nature. Here empathy level is examined in five different academic years. A prospective study is needed to follow students annually from the beginning of first year until graduation, to find out an accurate image of change in empathy levels. The present study only focuses on students attending a private medical college in Bangladesh. Larger study populations covering different medical colleges of Bangladesh are needed to validate the results of this study.

\section{Conclusion}

The results of this study provide support for the reliability and construct validity of the Bengali version of JSE Sversion. There were significant associations between gender and empathy scores. The level of empathy in medical students gradually increases after clinical training in medical college. It is suggested that the medical curriculum in Bangladesh should include more exercises and extensive program to promote empathy and other humanistic values among the medical students.

\section{Abbreviations}

\author{
JSE: $\quad$ Jefferson Scale of Empathy \\ CMOSHMC: Chattagram Maa-O-Shishu Hospital \\ Medical College \\ JSE S-version: Jefferson Scale of Empathy Medical \\ Student version.
}

\section{Conflict of Interests}

The authors declare that there is no conflict of interests regarding the publication of this paper.

\section{Acknowledgments}

The authors would like to thank Mohammadreza Hojat and Kaye Maxwell at Jefferson Medical College for kindly granting permission to use the JSE S-version in this study. They are also grateful to all the medical students of CMOSHMC who have voluntarily participated and extended their kind cooperation during data collection.

\section{References}

[1] "Empathy," Oxford Dictionaries, http://oxforddictionaries .com/definition/english/empathy.

[2] M. Hojat, J. S. Gonnella, and K. Maxwell, Jefferson Scales of Empathy (JSE): ProfeSSional Manual \& User's Guide, Jefferson Medical College-Center for Research in Medical Education and Health Care, Philadelphia, Pa, USA, 2009.

[3] S. D. Hodges and K. J. K. Klein, "Regulating the costs of empathy: the price of being human," Journal of Socio-Economics, vol. 30, no. 5, pp. 437-452, 2001.

[4] J. Shapiro, "Walking a mile in their patients' shoes: empathy and othering in medical students' education," Philosophy, Ethics, and Humanities in Medicine, vol. 3, no. 1, article 10, 2008.

[5] K. M. Glaser, F. W. Markham, H. M. Adler, R. P. McManus, and M. Hojat, "Relationships between scores on the Jefferson Scale of physician empathy, patient perceptions of physician empathy, and humanistic approaches to patient care: a validity study," Medical Science Monitor, vol. 13, no. 7, pp. 291-294, 2007.

[6] M. Hojat, J. S. Gonnella, S. Mangione et al., "Empathy in medical students as related to academic performance, clinical competence and gender," Medical Education, vol. 36, no. 6, pp. 522-527, 2002.

[7] İ. Gönüllü and D. Öztuna, "Turkish Adaptation of the Student Version of the Jefferson Scale of Physician Empathy," Marmara Medical Journal, vol. 25, pp. 87-92, 2012.

[8] M. Hojat, S. Mangione, T. J. Nasca et al., "An empirical study of decline in empathy in medical school," Medical Education, vol. 38, no. 9, pp. 934-941, 2004.

[9] M. Hojat, M. J. Vergare, K. Maxwell et al., "The devil is in the third year: a longitudinal study of erosion of empathy in medical school," Academic Medicine, vol. 84, no. 9, pp. 1182-1191, 2009.

[10] D. Chen, R. Lew, W. Hershman, and J. Orlander, "A crosssectional measurement of medical student empathy," Journal of General Internal Medicine, vol. 22, no. 10, pp. 1434-1438, 2007.

[11] E. Magalhães, A. P. Salgueira, P. Costa, and M. J. Costa, "Empathy in senior year and first year medical students: a crosssectional study," BMC Medical Education, vol. 11, no. 52, 2011. 
[12] P. J. Rosenfield and L. Jones, "Striking a balance: training medical students to provide empathetic care," Medical Education, vol. 38, no. 9, pp. 927-933, 2004.

[13] K. Vallabh, "Psychometrics of the student version of the Jefferson Scale of Physician Empathy (JSPE-S) in final-year medical students in Johannesburg in 2008," South African Journal of Bioethics and Law, vol. 4, no. 2, 2011.

[14] S. Tavakol, R. Dennick, and M. Tavakol, "Psychometric properties and confirmatory factor analysis of the Jefferson Scale of Physician Empathy," BMC Medical Education, vol. 11, no. 1, article 54, 2011

[15] H. U. Kataoka, N. Koide, K. Ochi, M. Hojat, and J. S. Gonnella, "Measurement of empathy among Japanese medical students: psychometrics and score differences by gender and level of medical education," Academic Medicine, vol. 84, no. 9, pp. 11921197, 2009.

[16] M. S. Roh, B. J. Hahm, D. H. Lee, and D. H. Suh, "Evaluation of empathy among Korean medical students: a cross-sectional study using the Korean version of the Jefferson scale of physician empathy," Teaching and Learning in Medicine, vol. 22, no. 3, pp. 167-171, 2010.

[17] M. Rahimi-Madiseh, M. Tavakol, R. Dennick, and J. Nasiri, "Empathy in Iranian medical students: a preliminary psychometric analysis and differences by gender and year of medical school," Medical Teacher, vol. 32, no. 11, pp. 471-478, 2010.

[18] M. Hojat, J. S. Gonnella, S. Mangione, T. J. Nasca, and M. Magee, "Physician empathy in medical education and practice: experience with the Jefferson scale of physician empathy," Seminars in Integrative Medicine, vol. 1, no. 1, pp. 25-41, 2003.

[19] J. Kliszcz, K. Nowicka-Sauer, B. Trzeciak, P. Nowak, and A. Sadowska, "Empathy in health care providers-validation study of the Polish version of the Jefferson Scale of Empathy," Advances in medical sciences, vol. 51, pp. 219-225, 2006.

[20] H. B. Paro, R. M. Daud-Gallotti, I. C. Tibério, R. M. C. Pinto, and M. A. Martins MA, "Brazilian version of the Jefferson Scale of Empathy: psychometric properties and factor analysis," $B M C$ Medical Education, vol. 12, no. 73, 2012.

[21] M. Hojat, S. Mangione, T. J. Nasca et al., "The Jefferson Scale of Physician Empathy: development and preliminary psychometric data," Educational and Psychological Measurement, vol. 61, no. 2, pp. 349-365, 2001.

[22] D. Wen, X. Ma, H. Li, Z. Liu, B. Xian, and Y. Liu, "Empathy in Chinese medical students: psychometric characteristics and differences by gender and year of medical education," BMC Medical Education, vol. 13, no. 130, 2013.

[23] H. Kataoka, N. Koide, M. Hojat, and J. S. Gonnella, "Measurement and correlates of empathy among female Japanese physicians," BMC Medical Education, vol. 12, no. 48, 2012.

[24] S. M. Kongsved, M. Basnov, K. Holm-Christensen, and N. H. Hjollund, "Response rate and completeness of questionnaires: a randomized study of internet versus paper-and-pencil versions," Journal of Medical Internet Research, vol. 9, no. 3, article e25, 2007.

[25] D. George and P. Mallery, SPSS for Windows Step by Step: A Simple Guide and Reference, 11.0 Update, Allyn \& Bacon, Boston, Mass, USA, 4th edition, 2003.

[26] H. F. Kaiser, "The application of electronic computers to factor analysis," Educational and Psychological Measurement, vol. 20, pp. 141-151, 1960.

[27] B. Morling and M. Lamoreaux, "Measuring culture outside the head: a meta-analysis of individualism-collectivism in cultural products," Personality and Social Psychology Review, vol. 12, no. 3, pp. 199-221, 2008.

[28] S. Hasan, N. Al-Sharqawi, F. Dashti et al., "Level of Empathy among Medical Students in Kuwait University, Kuwait," Medical Principles and Practice, vol. 22, pp. 385-389, 2013. 


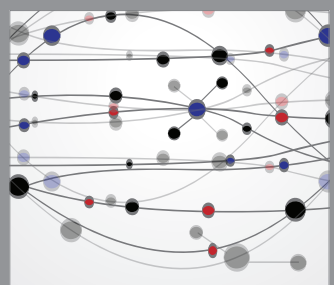

The Scientific World Journal
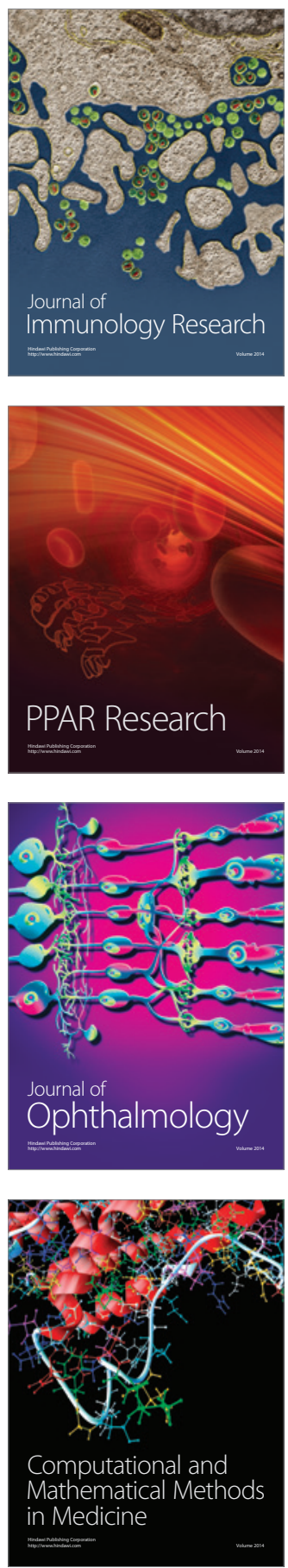

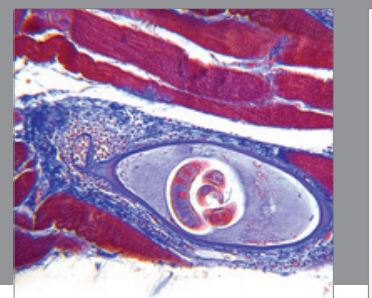

Gastroenterology

Research and Practice
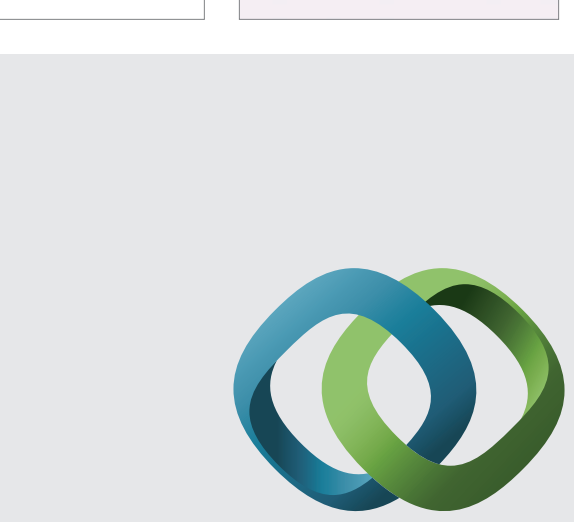

\section{Hindawi}

Submit your manuscripts at

http://www.hindawi.com
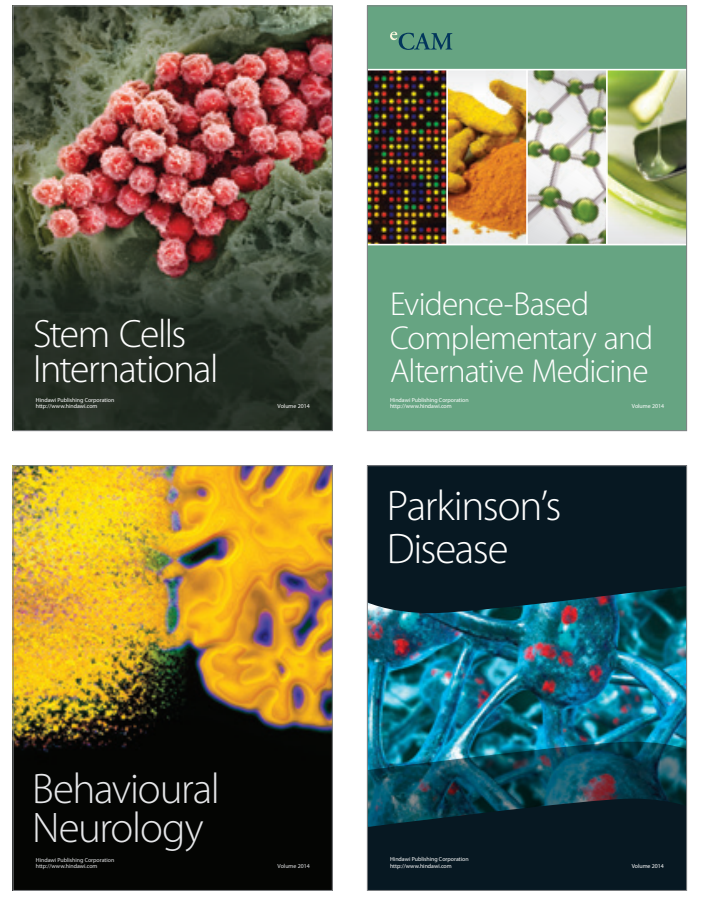
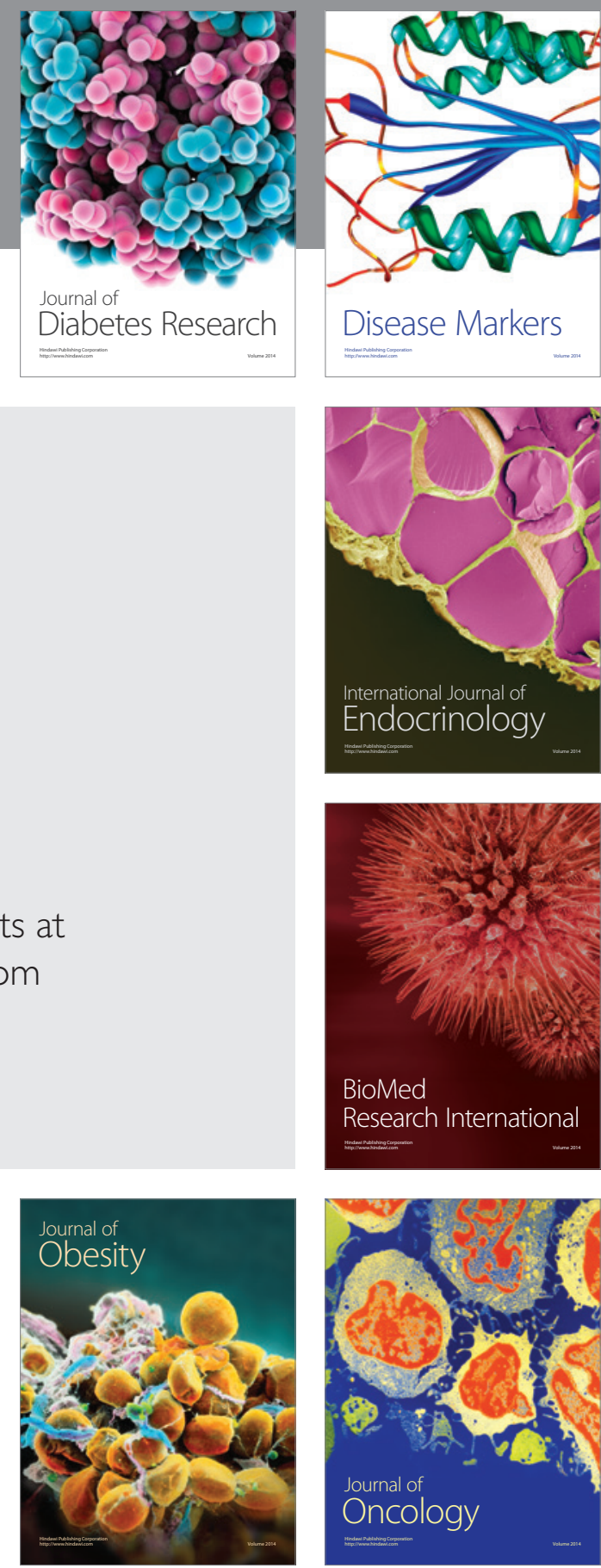

Disease Markers
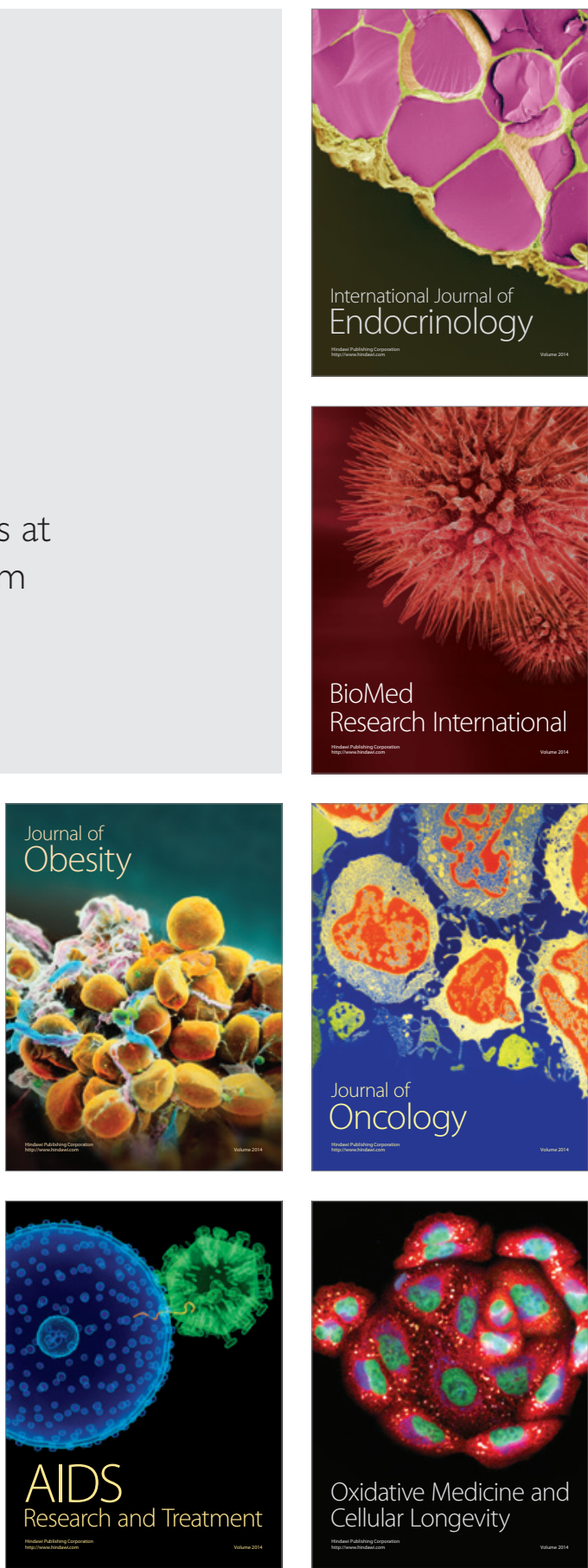